\title{
E-DROP: An Effective Drop Buffer Management Policy for DTN Routing Protocols
}

\author{
Sulma Rashid, Qaisar Ayub, M. Soperi Mohd Zahid, A.Hanan. Abdullah \\ Faculty of Computer Science \& Information System, \\ Department of Computer System \& Communication \\ Universiti Teknologi Malaysia (UTM) Skudai - Johor, 81310, Malaysia.
}

\begin{abstract}
In Delay Tolerant Networks (DTN) disruptions may happen frequently as end to end path is not available all the time. Thus, delays can also be extended due to its environment nature like deep space, underwater, ocean sensor networks. In manage to achieve message delivery probability in such demanding networking situations, researchers have proposed the design of store-carry-and-forward routing protocols, here a node might accumulate a message in its buffer and carry it next to for unlimited time, awaiting till a suitable forwarding opportunity acquire .Moreover, multiple message duplication into the network to increase delivery probability. This arrangement of long-standing storage and replication force a high storage overhead on network. Therefore, efficient buffer drop policies are required to resolve on buffer, which decides messages must be dropped, while node buffers are overflow.

In this paper, we propose effective buffer management drop policy E-DROP for delay tolerant networks. We illustrate that conventional buffer management policy like MOFO be unsuccessful to consider all relevant information in this framework. E-DROP policy can be adjust to minimize the metrics of relayed, dropped, average latency, overhead ratio ,hop count and to maximize the average delivery probability and buffer time. Using simulations support on an imitation mobility models shortest path map based and Map route movements, we show that our buffer management E-DROP with random message sizes drop policy performs better as the existing MOFO.
\end{abstract}

\section{General Terms}

Algorithm, Routing.

\section{Keywords}

Store and carry networks; Drop policy; routing.

\section{INTRODUCTION}

Recognition to the ever-increasing number of mobile devices with wireless abilities [7], the opportunity of communication lacking network infrastructure is becoming a reality. For instance, pocket switched networks [12] rely entirely on users exchange messages in the middle of themselves upon immediacy encounters. Present that a significant amount of traffic is composed of delay tolerant messages, this instance, known also as delay tolerant networks (DTN) [8], could supply to extensively reduce infrastructure costs and increasing bandwidth by orders of extent [9] and not confirmed end to end path as in the other tradition network TCP/IP.
In spite of a large amount of effort spend in the design of efficient routing algorithms for DTNs; there has not been an alike focal point on drop policies. Until now, the combination of durable storage and the, regularly expensive, message replication performed by many DTN routing protocols [3, 4] impose a high bandwidth and storage overhead on wireless nodes [10]. Furthermore, the data units dispersed in this context, called bundle, are independent.

In DTN Application-level data units which can be big [11]. It is obvious so as to, in this framework, node buffers will very probable run out of capability to store message for long time. For the alike causes, when mobility consequences in small time duration links between nodes, on hand bandwidth might be inadequate to communicate all intended messages. As a result, well-organized drop policies are necessary to make a decision

Which message(s) ought to be discarded when a node's buffer is occupied, mutually with competent preparation policies to decide which messages should be selected when bandwidth is partial, apart from of the precise routing algorithm selected?

As demonstrate by [13] that multi copy policy has high impact on message delivery and robustness at the cost of more bandwidth, energy and memory usage. However an important issue which was not investigated in the previous work is the use of an efficient buffer management strategies and message forwarding polices. A recent work [14], [15], [16], [18], [19] and [20] have proposed few buffer drop management strategies under congestion environments.

In this paper we introduce the buffer drop strategy E-DROP with to optimize the performance of DTN routing protocols in term of relayed, dropped, delivery probability, latency time averages, overhead ratio, hop count averages and buffer time averages.

The rest of paper is set as follows, Section 2 present existing buffer drop policies. Section 3 discusses router under observation, performance metrics are in Section 4, Section 5 depicts approach and E-DROP algorithm while section 6 is about simulation setup and results with conclusion in section 7 .

\section{EXISTING BUFFER MANAGEMENT POLICES}

\subsection{Drop Random (DR)}

The selection of message to be dropped is in hit and misses order. 


\subsection{Drop -Least-Recently-Received (DLR)}

The message with the long stay time in buffer will be dropped. The idea is that the packet with in buffer for lengthy time has less probability to be conceded to other nodes.

\subsection{Drop-Oldest (DOA)}

The message with the shorted remaining life time (TTL) in network will be selected to drop. The idea of dropping such packet is that if packet TTL is small, it is in the network for long time and thus has high probability to be previously delivered.

\subsection{DL-Drop last (DL)}

It drops the recently received message.

\subsection{Drop front (DF)}

The message that go into first in the queue is to be selected to drop first.

\subsection{N-Drop}

In N-Dropt [15], the message that achieves $\mathrm{N}$ number of forwarding will be selected to drop.

\subsection{Drop Larges (DLA)}

In Drop Largest (DLA), big size message will be selected in order to drop. [16]

\subsection{MOFO - Evict most forwarded first}

The message that has been forwarded to maximum number of times will be dropped first. [14]

\subsection{MOPR - Evict most favorably forwarded first}

Each message in node is related with a forwarding predictability $\mathrm{FP}$, initially assigned to 0 . When the message is forwarded the FP value is modified and the message with maximum FP value will be dropped first. [14]

\subsection{SHLI - Evict shortest life time first}

The message contain smallest TTL will be selected to drop. [14]

\subsection{LEPR - Evict least probable first}

"Since the node is minimum amount likely to deliver a message for which it has a low P-value. Drop the message for which the node has the lowest $P$ value." [14]

\subsection{GBD (Global Knowledge based Drop)}

GBD based on global knowledge about the network state. As global Knowledge is required, GBD is difficult to be implemented, thus, it will serve as a point of reference. [17]

\subsection{HBD}

A deployable variant of GBD that uses the new utilities based on estimates of $m$ and $n$. [17]

\subsection{FBD (Flood Based Drop)}

FBD accounts only for the global information collected using simple message flooding, that is, without considering past history or other messages. [17]

\subsection{T-DROP}

Its will drop the message which lies in the threshold message size range of buffer. [18]

\section{ROUTING PROTOCOLS FOR EVALUATIONS}

\subsection{Epidemic}

Epidemic routing [3] is a flooding multi-copy routing scheme which repeatedly used as a baseline for evaluation for the reason that, while tremendously inefficient in the number of forwards, it is optimal in terms of delivery probability. Moreover, Epidemic routing is very sufficient when investigate equality, as its load allocation is originally only by the network topology irrespective it does not be use any heuristic to direct the forwarding.

\subsection{Direct delivery (DD)}

The source node [2] sends out the message to other node only when other node is its destination. Direct delivery can be measured a mixture method as one can comprise it in flooding where Direct Delivery always select the direct path between source and destination.

\subsection{First Contact (FC)}

In first contact a message is forwarded along a single path by selecting the node randomly from available links. If connections do not exist the nodes waits and transmit the message to first available contact.

In first contact [1], [2] routing, the router transmits the message randomly as other nodes come under its communication range. Under congested networks the messages can not route.

\subsection{PROPHET}

PROPHET [4] is widely used as the orientation algorithm in terms of efficiency (e.g. in [5], [6]). PROPHET uses the history of contacts to analyze the probability of a node to send the message to their destination.

\section{PERFORMANCE METRICS}

\subsection{Relayed}

Relayed is ratio of message send to other nodes still reaching to destination?

\subsection{Dropped Ratio}

Drop ratio is function to count the messages dropped during transmission 


\subsection{Delivery probability}

It is the ratio of message received over message send. High probability means that more messages are delivered to the destination.

\subsection{Overhead-ratio}

It is the negation of number of messages relayed to number of message delivered. Low value of overhead means less processing required delivering the relayed messages. Objective of algorithm is to minimize the value of overhead.

\subsection{Latency Time Average}

Latency is that contributes to network speed. The term latency refers to any of several kinds of delays typically incurred in processing of network data. A supposed low latency network link is one that normally small delay times, whereas a high latency link usually experiences from long delays .in DTN latency is high due to its network nature.

\subsection{Buffer time average}

It is Sum of time spend by 1 message(s) in buffer divided by message send.

\subsection{Hop count average}

It is the Mean hops which a message takes to reach its destination.

\section{APPROACH}

\subsection{A: Scenario MOFO -Drop Sequence}

Let node A, M, R and D be messages, relay and drop sequence respectively. Node A buffer holds M list \{M1, M2, M3, M4, M5, M6 $\}$ at time $t$ with Sizes $S M=\{100,100,200,100,600,110\}$ and number of forwarding NOF as shown in Figure 1 . holding message Node B $\in\{$ M8,M21,M32,M43,M75,M90 $\}$.when both nodes $\mathrm{A}, \mathrm{B}$ come in the transmission time of each other their intermitting time $\lambda$ is the message rate between two nodes $\lambda=1 / \mathrm{E}[\mathrm{U}]$ where $\mathrm{E}[\mathrm{U}]$ is the average meeting time. M $8 \in$ Node B is R message transmit to Node A. As we see that Node A is congested and its selected messages with $\mathrm{D}$ sequence is $\mathrm{MOFO}$ .According to MOFO the D of $\mathrm{A}=\{\mathrm{M} 6, \mathrm{M} 3, \mathrm{M} 2, \mathrm{M} 4, \mathrm{M} 1, \mathrm{M} 5\}$. Therefore node A will drop $\{$ M6, M3, M2, M4, M1 $\}$ messages to accommodate M8. This technique gives more messages from buffer and cause more overhead.
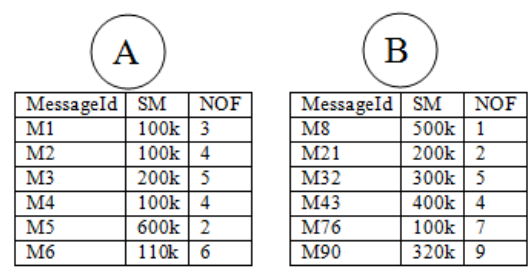

Figure 1 MOFO Snapshot

\subsection{B: Scenario E-Drop -Drop Sequence}

Figure 2 depicts the drop of message with E drop (Equal drop), where the $\mathrm{M}$ will be dropped $\mathrm{D}$ only if its size fall with in an equal or greater than incoming message otherwise no drop. D sequence will be sorted list descending to incoming message

When Node B transmits the message M8, node A will check its queue for available buffer space. As we can see that the queue of Node A is congested and it needs to drop a message. Assume the equal drop it's select the message equal and greater its finds M5 to drop for the accommodation of M8. This technique give high buffer time to small messages results in less message drop with high delivery probability.
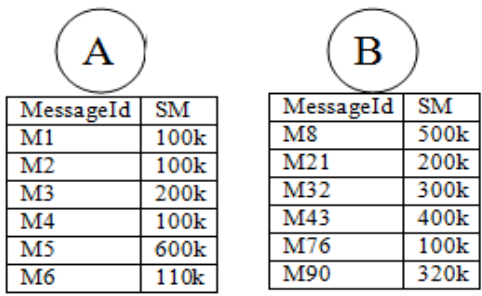

Figure 2 E-DROP Snapshots

\section{ALGORITHM}

Table 1: Variables

\begin{tabular}{|c|c|}
\hline Variable & Description \\
\hline $\mathrm{i}$ & Number of $\mathrm{i}$ node $\mathrm{i}=\{1,2,3 \ldots \mathrm{n}\}$ \\
\hline $\mathrm{B}_{\mathrm{NA}}$ & Buffer available \\
\hline $\mathrm{M}_{\mathrm{N}}$ & New message \\
\hline $\mathrm{M}_{\mathrm{R}}$ & Messages currently help by Node \\
\hline SN & Size of new Message \\
\hline \multicolumn{2}{|c|}{ I* drop equal message to make room for new message* } \\
\hline $\begin{array}{l}\text { While }( \\
\text { doubl } \\
\text { BNA } \\
\text { delet }\end{array}$ & $\begin{array}{l}\left.>=\mathrm{B}_{\mathrm{NA}}\right) \\
\text { =CALL getEqualMessage(SN) } \\
\text { BNA + MessageCollection.get(id).Size(); } \\
\text { essage(MessageCollection.get(id)); }\end{array}$ \\
\hline
\end{tabular}

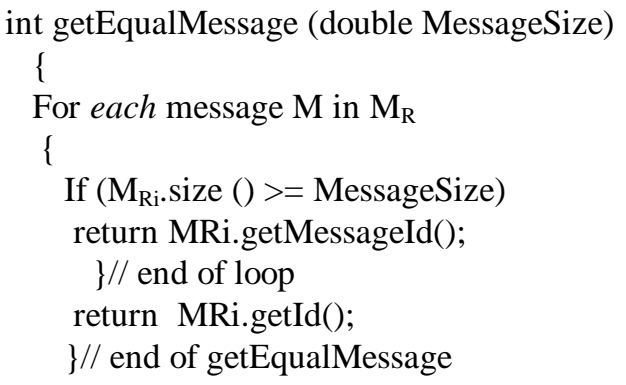




\section{SIMULATIONS AND RESULTS}

In the following section we have examined routing protocols (Section 3), with exiting (MOFO) and proposed (E-DROP).

All the experiments were carrying out by ONE Simulator. The ONE Simulator is a discrete event simulator written in Java. The major aspire of simulator is to relate DTN (store-carry-forward) of message for long time, where the probability of disconnections and failures enlarged.

Table 2 Simulation parameters

\begin{tabular}{|l|l|}
\hline Number of Nodes & 126 \\
\hline Movement model 1 & $\begin{array}{l}\text { Shortest Path Map based } \\
\text { Movement }\end{array}$ \\
\hline Movement model 2 & Map rout movement \\
\hline $\begin{array}{l}\text { Number of groups } \\
\text { Model 1+2 }\end{array}$ & $03+03$ \\
\hline Buffer size of Model 1+2 & $6 \mathrm{MB}+50 \mathrm{MB}$ \\
\hline Transmission range & $10 \mathrm{M}$ \\
\hline Transmission speed & $250 \mathrm{~K}$ \\
\hline Message creation interval & $200-300$ (seconds) \\
\hline Messages size randomly & $500 \mathrm{k}-1 \mathrm{M}$ \\
\hline Simulations times varies & $200000 \mathrm{~s}$ \\
\hline Area of Simulation & $4500 \mathrm{mx} 3400 \mathrm{~m}$ \\
\hline
\end{tabular}

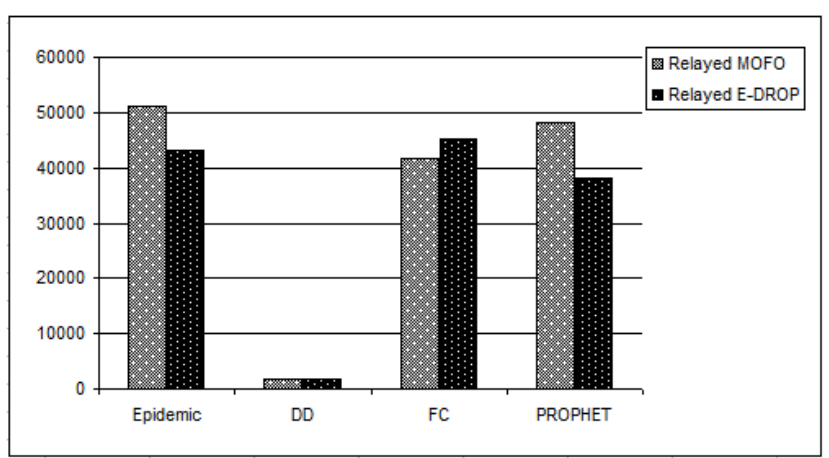

Figure 3 E-DROP, MOFO Relayed w.r.t Routers

The above Figure 3 reveals the comparison of MOFO and EDROP queue drop polices with respect to relayed messages. In all router configurations E-DROP reduces the message relayed to a significant quantity. We can observe the strength of E-DROP, which drop equal size messages and free less buffer space as described in algorithm (section 4) and retain most of the message to overcome from waste resources(more relays). In FC, the message relayed was little increased which is good for single copy scheme of routing due to its routing algorithms randomly selection for next hop and attain more delivery of messages. In $\mathrm{DD}$, the relayed message are equal because its deliver the message on the bases on destination encounter else it retain the message in E-DROP. Hence as compared to existing MOFO, EDROP has optimized the message relayed metric

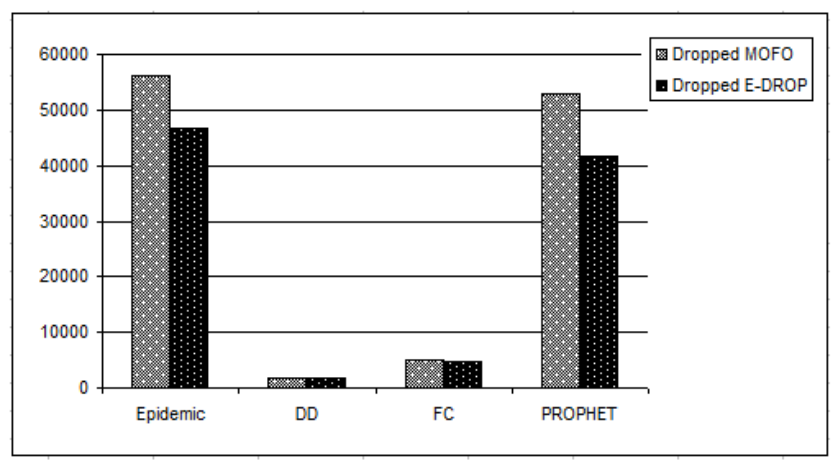

Figure 4 E-DROP, MOFO Dropped w.r.t Routers

Figure 4 plots the impact of message dropped with existing MOFO and with our drop policy E-Drop. In all router configurations reduce the message drops to a significant quantity. We can observe the strength of E-Drop, which drop equal size message and free those buffer messages whom are equal to incoming message size resulted less drop as described in algorithm (section 4).Our E-drop is better for multi and single copy routing. In DD its equal due to its routing algorithms approach but FC it's reduce drop and deliver more messages to its destination. Comparable from MOFO, E-Drop has optimized the message drop metric in a reasonable level.

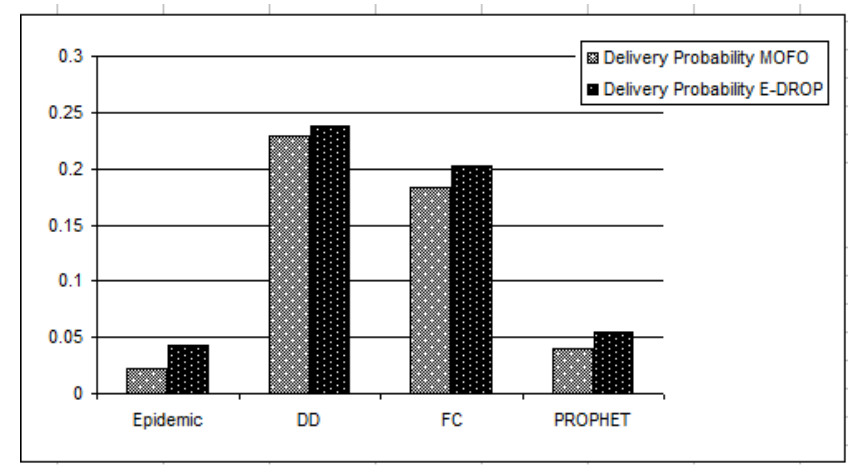

Figure 5 E-DROP, MOFO Delivery Probability w.r.t Routers

The above Figure 5 depicts the comparison of MOFO and EDROP queue drop policy with respect to delivery probability. We can examine that epidemic, prophet, FC,DD routing protocols have increase the delivery probability because frequency of node encounter is high resulting more congestion where E-drop drop message in case of equal size of message as the new message will come and raise the delivery of messages. FC and DD are single copy cases and chance of encounter is less as in multi copy schemes result E-drop cases suits single copy case resulted increases in delivery probability as compared to other routers but still MOFO increases the delivery than existing queue policy. Moreover, DD router passes the messages to that node that are destination. However in all the configuration of routers, message delivery probability of E-DROP is improved then MOFO. 


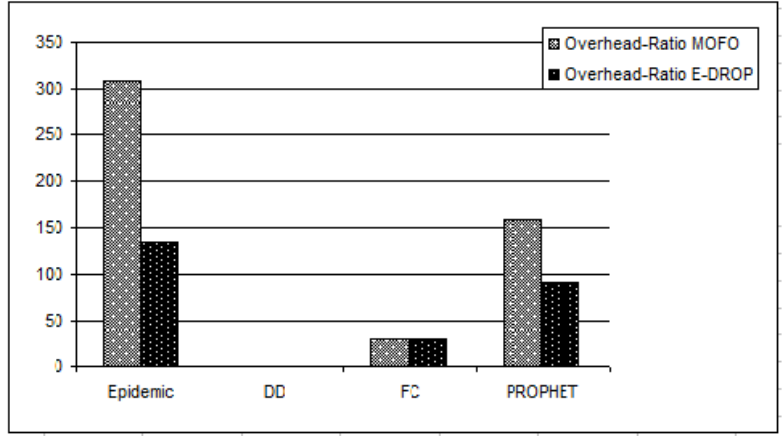

Figure 6 E-DROP, MOFO overhead ratio w.r.t Routers

Figure 6 represents the affects of MOFO and E-DROP with respect to overhead ratio. We are able to notice clearly that overhead ratio with E-DROP is decreases in all routers irrespective of multi copy or single copy approaches of routers. DD due to direct transmission overhead is zero so we excluded that case for both algorithms, while Epidemic, , Prophet, FC overhead is reduced to considerable level because our E-drop policy selected equal size or nearly grater sizes of message which dropped less message from node buffer and that why overhead decrease in a huge amount as compared to MOFO.

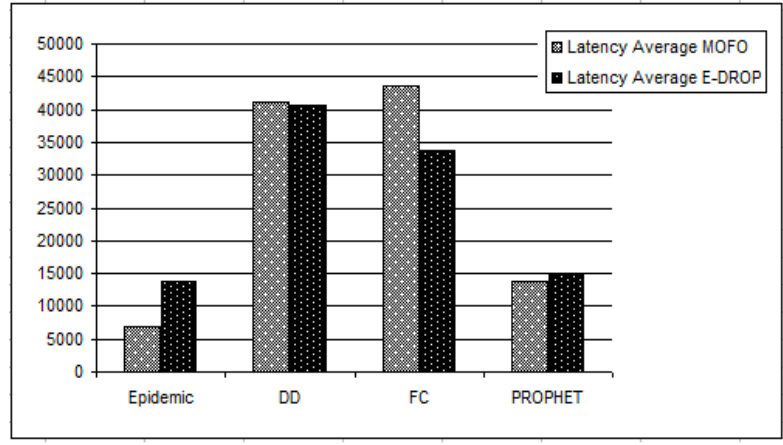

Figure 7 E-DROP, MOFO Latency averages w.r.t Routers

Figure 7 shown the influence of MOFO and E-DROP of routers with respect to Latency averages. We are able to become aware that Latency with E-DROP is decreases in FC and DD routers respective single copy approaches of routers. DD due to direct transmission latency is almost equal; while Epidemic and Prophet Latency averages are increases due to flooding nature and multi copy cases.

In Figure 8 we simulated the result of MOFO and E-DROP for Epidemic FC, DD and prophet router under hop count average. The increase in hop count reflects the fact that message has consumed more resources to reach its destination. The minimum value of hop count confirms less overhead and delivery delay.

We can observe that with E-DROP policy the hop count average for all routers have reduced at a constant ratio. In MOFO when ever congestion arise it continue to drops the messages to free space for new message resulting more relay and increase in hop

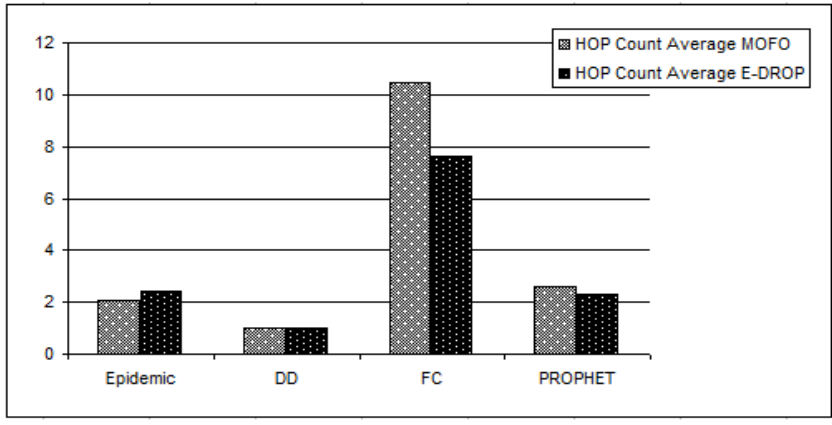

Figure 8 E-DROP, MOFO hop count average w.r.t Routers

count average. While in E-Drop stops this redundant unnecessary drop and provides prevention against unnecessary relay and overhead.

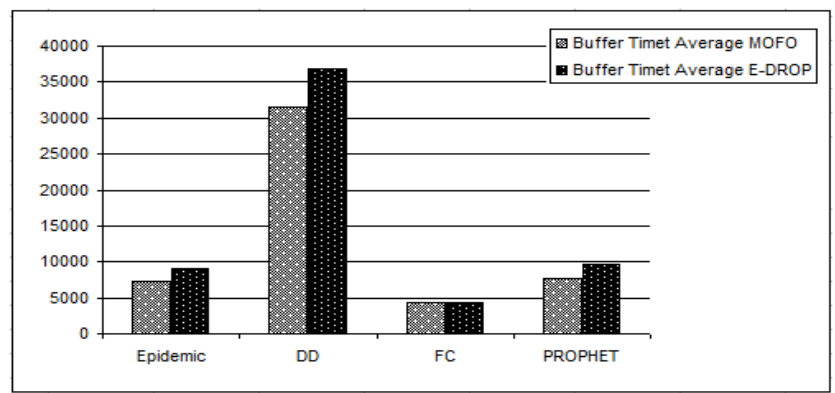

Figure 9 E-DROP, MOFO buffer time average w.r.t Routers

Figure 9 observes buffer time averages with E-DROP and MOFO. It can be clearly seen that E-DROP has high value of buffer time average with all router. Buffer time occupancy is very scared resource in the DTN where store and carry paradigms are used. As expected, in this architecture buffer should retain the message as along as it can, so that delivery of message should increase and eliminate the drop ratio of messages. E-DROP improved the buffer time occupancy for all routers in multi and single copy routers and increases the delivery ratio as in the figure

\section{CONCLUSION}

In this work, it is investigated a local optimal buffer management drop strategy E-DROP under congestion by means of random sizes of messages and message interval with respect to DTN routing to optimize the performance of protocols in term of relayed, dropped, delivery probability, latency time averages, overhead ratio, hop count averages and buffer time averages.

With the aim of this work, we considered our comparison with MOFO drop policy. It would be motivating to examine it with other existing drop policies that take into explanation of different message sizes. The consideration of routing protocols other than Epidemic, DD, FC, and Prophet are also an interesting direction to survey.

\section{REFERENCES}

[1] KERÄNEN, A., AND OTT, J. Increasing Reality for DTN Protocol Simulations. Tech. rep., Helsinki University of Technology, Networking Laboratory, July 2007.

[2] SPYROPOULOS, T., $\quad$ PSOUNIS, K., AND 
intermittently connected mobile networks. In Proc. Sensor and Ad Hoc Communications and Networks SECON (October 2004), pp. 235-244.

[3] Vahdat and D. Becker, "Epidemic routing for partially connected ad hoc networks," Duke University, Tech. Rep. CS-200006, 2000.

[4] Lindgren, A. Doria, and O. Schelen. Probabilistic Routing in intermittently connected networks, volume 3126, pages 239-254. 2004.

[5] E. Daly and M. Haahr. Social network analysis for routing in disconnected delay-tolerant manets. In Proc. of ACM MobiHoc, 2007.

[6] P. Hui, J. Crowcroft, and E. Yoneki. Bubble rap: Social based forwarding in delay tolerant networks. In Proc. of MobiHoc, 2008.

[7] S. Keshav. Why cell phones will dominate the future internet. SIGCOMM Comput. Commun. Rev., 35(2):83-86, 2005

[8] K. Fall. A delay-tolerant network architecture for challenged internets. In Proc. SIGCOMM, 2003.

[9] M. Grossglauser and D. Tse. Mobility increases the capacity of ad hoc wireless networks. IEEE/ACM Transactions on Networks, 10(4):477-486, 2002.

[10] T. Spyropoulos, K. Psounis, and C. S. Raghavendra. Efficient routing in intermittently connected mobile networks: The multiple-copy case. ACM/IEEE Transactions on Networking,Feb. 2008.

[11] Delay tolerant networking research group. http://www.dtnrg.org.

[12] P. Hui, A. Chaintreau, J. Scott, R. Gass, J. Crowcroft, and C. Diot,"Pocket switched networks and human mobility in conference environments,"in Proc. ACM SIGCOMM Workshop on Delay Tolerant Networking(WDTN), 2005.

[13] T. Small and Z. J. Haas. Resource and performance tradeoffs in delay-tolerant wireless networks. In
SIGCOMM Workshop on Delay Tolerant Networking (WDTN), 2005.

[14] A.indgren and K. S. Phanse, "Evaluation of queuing policies and forwarding strategies for routing in intermittently connected networks,"in Proc. of IEEE COMSWARE, pp. 1-10, Jan. 2006.

[15] Yun Li, Ling Zhao ,Zhanjun Liu,Qilie Liu." N-Drop Congestion Control strategy under Epidemic Routing in DTN." Research center for wireless information networks,chongqing university of posts \& Telecommunications ,chongqing 400065, china, pp. 457460, 2009

[16] Sulma Rashid,Qaisar Ayub,"Effective buffer management policy DLA for DTN routing Protocals under congetion", International Journal of Computer and Network Security, Vol 2,NO 9,Sep 2010. pp .118-121

[17] Amir Krifa, Chadi Barakat, Thrasyvoulos Spyropoulos, "Optimal Buffer Management Policies for Delay Tolerant Networks," IEEE Conference on Sensor, Mesh and Ad Hoc Communications and Networks (SECON 2008), June 2008.

[18] Qaisar Ayub, Sulma Rashid," T-Drop: An optimal buffer management policy to improve QOS in DTN routing protocols", Journal of Computing, Vol 2, ISSUE 10, OCTOBER 2010. pp .46-50.

[19] Sulma Rashid, Qaisar Ayub, M. Soperi Mohd Zahid , A.Hanan. Abdullah. "Optimization of DTN routing protocols by using forwarding strategy (TSMF) and queuing drop policy (DLA)", International Journal of Computer and Network Security, Vol 2,ISSUE 10, OCTOBER 2010. pp .71-75.

[20] Qaisar Ayub, Sulma Rashid and Dr.Mohd Soperi Mohd Zahid. Article:Optimization of Epidemic router by new forwarding queue mode TSMF. International Journal of Computer Applications 7(11):5-8, October 2010. Published By Foundation of Computer Science

Rochester Institute of Technology, New York and B.S. degree in 1988 from New Mexico State University, Las Cruces in Computer Science and Mathematics. He is currently a faculty member in Computer Science, Universiti Teknologi Malaysia. His research interests include Internet Routing Protocols and Delay/Disruption Tolerant Networks.

Professor Dr Abdul Hanan Abdullah is the Dean, Dept. of Computer Systems \&Communication, University Teknologi, Malaysia. He received the B.Sc. and M.Sc from University of San Francisco, California, and the Ph.D degree from Aston University,Birmingham, UK, in 1995. His research interest is in Information Security. $\mathrm{He}$ is also a head of Pervasive Computing Research Group (PCRG) UTM and member of $\mathrm{ACM}$
Science from University of Wisconsin, Milwaukee in 2009 , M.S. degree in Computer Integrated Manufacturing from 